\title{
A close-loop verification approach for pedestrian stability based on machine vision
}

\author{
Rongyong Zhao, Ping Jia", Yan Wang, Cuiling Li, Yunlong Ma, and Zhishu Zhang \\ School of Electronic and Information Engineering, Tongji University, Shanghai, China
}

\begin{abstract}
In public places, it is significant to analyze the stability of the crowd which can support the crowd management and control, and protect the evacuees safely and effectively. The numerical analysis method of system stability based on Lyapunov theory suffers problems that it is difficult to avoid random errors in the initialization of pedestrian density and velocity, as well as cumulative errors due to time increasing, limiting its application. This study adopts a complementary model of theoretical numerical analysis and machine vision with a parallel convolutional neural network $(\mathrm{CNN})$ model. It proposes an approach of stability analysis and closed-loop verification for crowd merging systems. Thereby, this research provides theoretical and methodological support for planning of the functional layout of crowd flow in public crowd-gathering places and the control measures for stable crowd flow.
\end{abstract}

Keywords: Machine vision, Stability analysis, A closed-loop verification.

\section{Introduction}

In recent years, the crowd flows in large-scale public places such as high-speed rail stations and airports in many parts of the world are increasing. Large-scale entertainment activities (concerts and light shows, etc.) increased, and stampede incidents occurred frequently, causing serious casualties and economic losses[1]. Most public places cover large areas with complex structures. They can accommodate thousands of tourists or pedestrians in a single crowd. These crowds are highly dense. According to the stampede accident records occurred around the world, many countries experienced stampede accidents to different extends.

Most stampede incidents stem from emergencies or panic incidents, and the crowd is stimulated. Crowds gather in a certain area for a short period and crowds are extremely prone to collisions during crowd movement, causing stampede incidents. At the same time, during crowd movement, the crowd density in a specific area is probably high, exceeding the safe range [2]. Nowadays, more scientific and effective methods to detect crowd density and maintain the stability of crowd movement are urgently needed, prevent excessive crowd density in public places, and prevent and avoid stampede accidents. Therefore,

*Corresponding author: 2033108@tongji.edu.cn 
maintaining the stability of crowd flow in public places and ensuring the safe and orderly movement during the evacuation of crowd in public places have become the one of hotspots of security researchers from all over the world.

\section{Analysis of numerical stability based on Lyapunov}

The crowd density judgment method is classified into five states according to the crowd density, i.e., free flow, restricted flow, dense flow, and extraordinary flow, very dense flow and jammed flow[3]. The numerical analysis method of system stability based on Lyapunov's theory is categorized into three states, namely, crowd stability, crowd marginal stability, and crowd instability. Among them, crowd stability involves free crowd movement and restricted crowd movement, crowd critical stability involves dense crowd movement, and crowd instability involves very dense crowd movement and blocked crowd movement. We obtain the criteria for judging crowd stability based on crowd density. Then, the critical range of crowd acceleration in crowd evacuation is obtained, the crowd merging acceleration-critical density time lag model is established, and the stability criterion of acceleration vector based on Lyapunov's crowd regional flow is obtained, as shown in the following Eqs.(1)-(4).

$$
\begin{gathered}
\alpha_{\mathrm{v}}=\frac{1}{2 \tau}\left(1-\rho_{0} \sqrt{-2 \mathrm{~V}_{\mathrm{e}}^{\prime}\left(\rho_{0}\right) \tau}\right) \\
\frac{1}{2} \geq-\rho_{0}^{2} \mathrm{~V}_{\mathrm{e}}^{\prime}\left(\rho_{0}\right) \tau \\
|| \mathrm{v}^{\prime} \|=\sqrt{\mathrm{V}_{\mathrm{e}}^{\prime 2}+\mathrm{U}_{\mathrm{e}}^{\prime 2}} \leq \frac{1}{2 \rho_{0}^{2} \tau} \\
\theta=\arctan \frac{\mathrm{U}_{\mathrm{e}}^{\prime}}{\mathrm{V}_{\mathrm{e}}^{\prime}}
\end{gathered}
$$

Among them, $\rho_{0}$ is the initial speed of the group, $\theta$ represents the direction of the group acceleration, $V_{e}$ and $U_{e}$ are the horizontal and vertical equilibrium speeds, respectively, and $\tau$ is the relaxation time coefficient, which is the time constant for adjusting the evacuation crowd speed to the equilibrium speed.

Taking $\frac{1}{2 \rho_{0}^{2} \tau}$ as the critical value, when the acceleration value of crowd movement exceeds the critical value $\frac{1}{2 \rho_{0}^{2} \tau}$, the crowd movement is in an unstable state, and extremely prone to collisions and even leads to serious stampede incidents[4]; When the crowd acceleration is less than the critical value, the crowd movement is in a stable state, which is conducive to safety protection in public places.

The Lyapunov system stability theory provides a simple and fast numerical calculation method for analyzing and predicting the stability of crowd merging[5]. The numerical analysis method of system stability based on Lyapunov theory can realize rapid prediction of crowd stability. On the other hand, the Lyapunov method also has the problems of crowd dynamics density, speed initialization error and random error accumulation. This method has inevitable parameter initialization errors and accumulated errors. Its calculation accuracy and precision are difficult to meet the requirements of the stability analysis of complex crowd merging. To this end, this study uses computer video and image processing technology to analyze the stability of the crowd in public places. A crowd density detection model based on convolutional neural network is proposed, and the crowd stability criterion is used to analyse and predict the crowd stability in the video in real time. 


\section{Machine vision based verification}

In the field of image processing, machine vision methods have high accuracy and highprecision performance in crowd counting and density estimation, and can support crowd crowding state assessment. In view of the existing Lyapunov theory analysis error defects, this study introduces the convolutional neural network $(\mathrm{CNN})$ model in the field of machine vision, collects the crowd data in the video surveillance image information, studies the crowd count and density estimation, and uses the density judgment method to analyze the crowd stability Sex [6]-[7]. Convolutional neural network is a special neural network model, which is specially used to process data with a similar grid structure. It performs well in image processing and computer vision applications. Convolution is a special linear operation. CNN uses convolution in some layers of the network instead of general matrix multiplication. This special operation method brings unique structural characteristics to $\mathrm{CNN}$, which helps to deepen the network level and enhance the ability of feature representation[8]. The main challenge of crowd counting is the different head sizes of people in the image due to the perspective effect. Therefore, by increasing the number of neural network columns with different convolution kernels, it is possible to extract head features of people of different sizes and ultimately improve the accuracy of recognition. In order to more fully extract the crowd features of different sizes in the image, improve the accuracy of crowd counting, and meet the requirements of high-precision detection of crowd density values, this paper uses a four-column convolutional neural network model $(4 \mathrm{C}-\mathrm{CNN})$ to solve the image crowd Counting problem.

\subsection{Machine vision and Lyapunov closed loop verification method}

Most stability analysis is mainly based on the numerical analysis of the disturbance numerical model and the system response. Parameters such as crowd speed and density produce initialization errors and accumulated errors, which affect the accuracy and precision of the stability numerical analysis. Therefore, how to combine the numerical analysis method of crowd stability with the high accuracy and precision method of crowd counting and density estimation in the field of machine vision, which not only exerts the rapid advantages of stability numerical analysis, but also exerts the high accuracy and precision of machine vision High-precision advantages, forming complementary advantages, closed-loop stability analysis and verification methods, have become the goal of this article. Aiming at the initialization errors and cumulative errors of parameters such as speed and density of Lyapunov stability analysis, a machine vision parallel convolutional neural network $(\mathrm{CNN})$ model was constructed to improve the accuracy and precision of target recognition in video data, and to verify the stability of channel crowd flow It also corrects the errors of numerical calculations regularly, and proposes a closed-loop verification method that complements the stability judgment theory and the actual measurement of machine vision.

The CNN model captures the pedestrian position and density at each moment ( $\mathrm{k}$ time step) with high accuracy and high precision, and returns it to the crowd dynamics model, verifies the stability conclusions of the numerical analysis with actual video collection data, and updates the grid The parameters such as the location and density of pedestrians in the area are corrected for the next moment ( $\mathrm{k}+1$ time step); The CNN model is used as the feedback link of the stability numerical analysis to obtain a closed-loop verification method that complements the crowd flow stability theory and the actual measurement. Machine vision is used to analyze the impact of factors such as disaster factors, confluence angle, number of pedestrians, crowd flow speed, grid area acceleration, crowd density, and crosschannel width ratio on crowd stability. Although machine vision methods cannot quickly 
predict crowd density, real-time calculations can compensate for the weaknesses of initialization and random errors in Lyapunov's stability analysis. Data feedback and parameter correction of real scenes are required to form a closed-loop verification of Lyapunov analysis theory and machine vision acquisition. Finally, according to the results of crowd stability, specific measures and suggestions for crowd safety management and control in this area are given, so as to prevent crowd trampling accidents in public places in time.

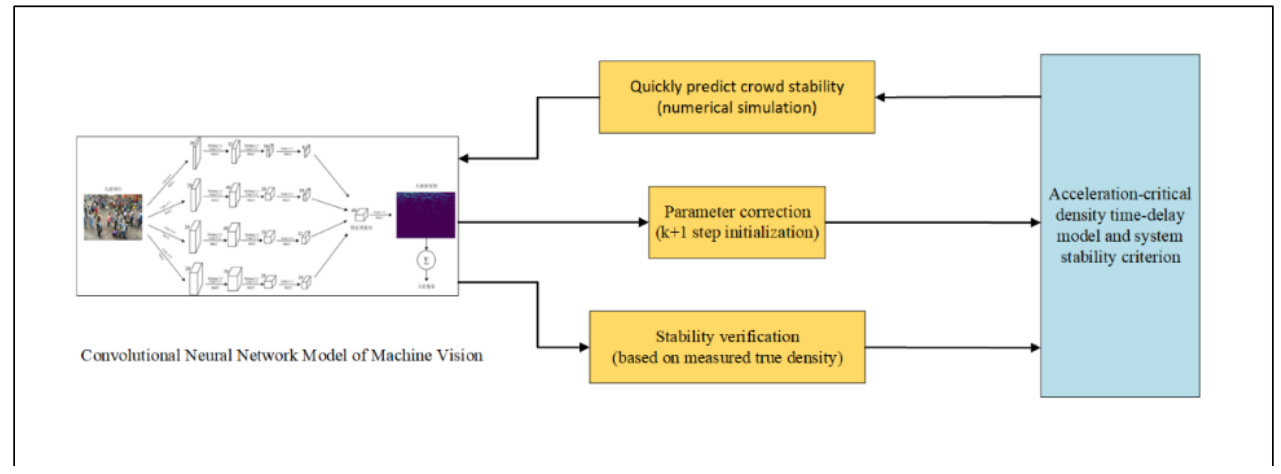

Fig. 1. Close-loop verification model for pedestrian stability based on machine vision.

\section{Case study}

This study takes the crowd stability based on machine vision in the waiting hall of Shanghai Hongqiao Railway Station as an example. Shanghai Hongqiao Railway Station is a component of Shanghai Hongqiao Comprehensive Transportation Hub and one of the important railway passenger transport hubs in East China. The huge passenger flow of Shanghai Hongqiao Railway Station brings huge challenges to safety management. Therefore, it is necessary to study the crowd density of Hongqiao Railway Station and predict the trend of crowd density and crowd stability. The actual scene of the waiting hall of Hongqiao Railway Station is shown in Figure 2.

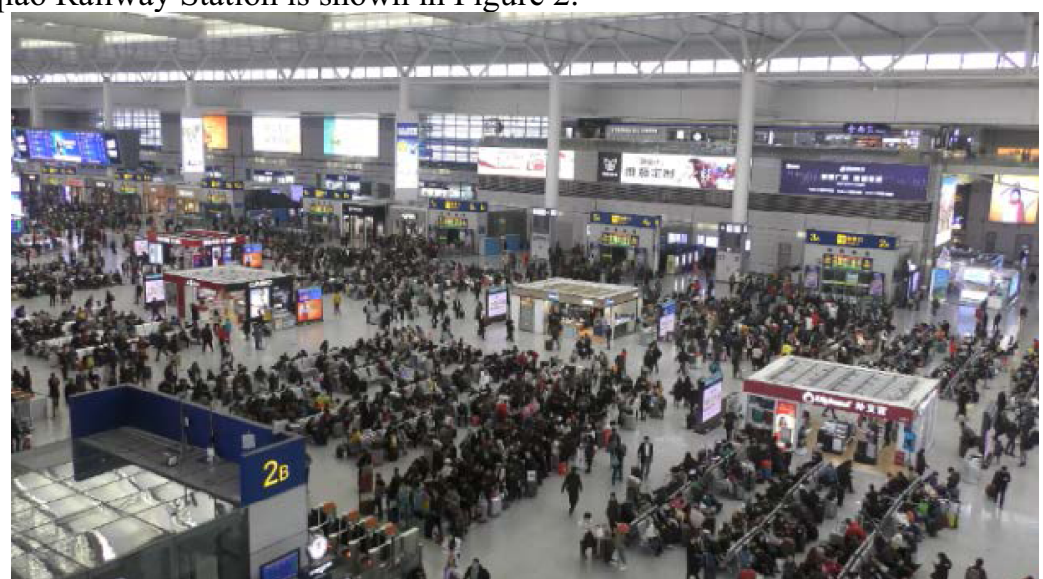

Fig. 2. Actual scene of the waiting hall of Shanghai Hongqiao Railway Station (captured on Dec.6th, 2019).

Through the crowd density detection model based on machine vision, the crowd density in the video is detected. We use the waiting hall of Shanghai Hongqiao Railway Station as the scene to obtain the number of people in a specific area (unit: $p$ ), and then combine the 
image correction technology to obtain the actual area of the area (unit: $\mathrm{m} 2$ ), and finally calculate the crowd density value in a specific area (unit: $\mathrm{p} / \mathrm{m} 2$ ). The crowd density distribution map of Shanghai Hongqiao waiting hall is obtained through simulation, and the maximum crowd density value is about $2 \mathrm{p} / \mathrm{m} 2$. The simulation display diagram is shown in Figure 3.

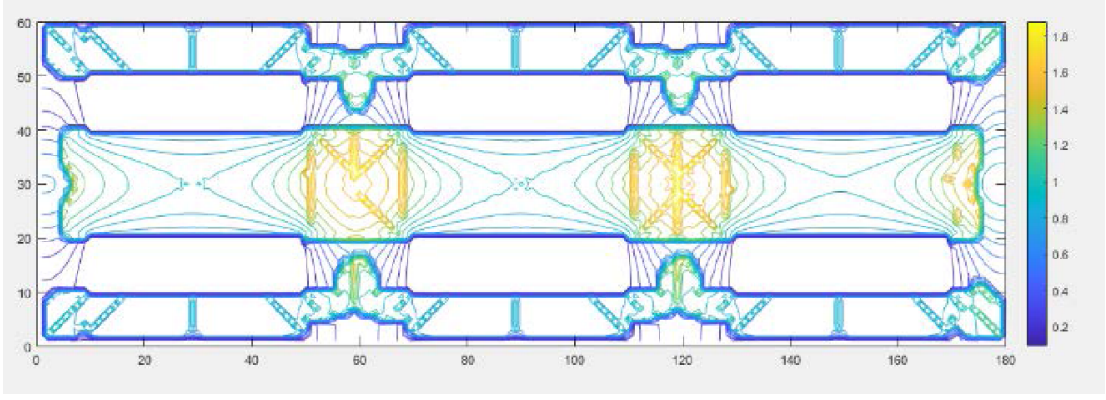

Fig. 3. Simulation map of crowd density in Shanghai Hongqiao waiting hall.

This study introduces the Mean Absolute Error (MAE) to use the data set to evaluate the effects of the two methods, which is defined as shown in formula (5). MAE, Mean Absolute Error, is the average value of the absolute value of the error between the observed value and the true value[9]. Among them, $x$ is the actual number of crowd, $x_{i}$ is the estimated number of crowd in the two methods, and $\mathrm{m}$ is the number of tests. The smaller the value of MAE, the higher the accuracy of the method.

$$
\operatorname{MAE}=\frac{1}{\mathrm{~m}} \sum_{\mathrm{i}=1}^{\mathrm{m}}\left|\mathrm{x}_{\mathrm{i}}-\mathrm{x}\right|
$$

The actual number of crowd in the waiting hall of Shanghai Hongqiao Railway Station is 3,000. Two methods are used for 50 tests, and the results are displayed as a line chart as shown in Figure 4.

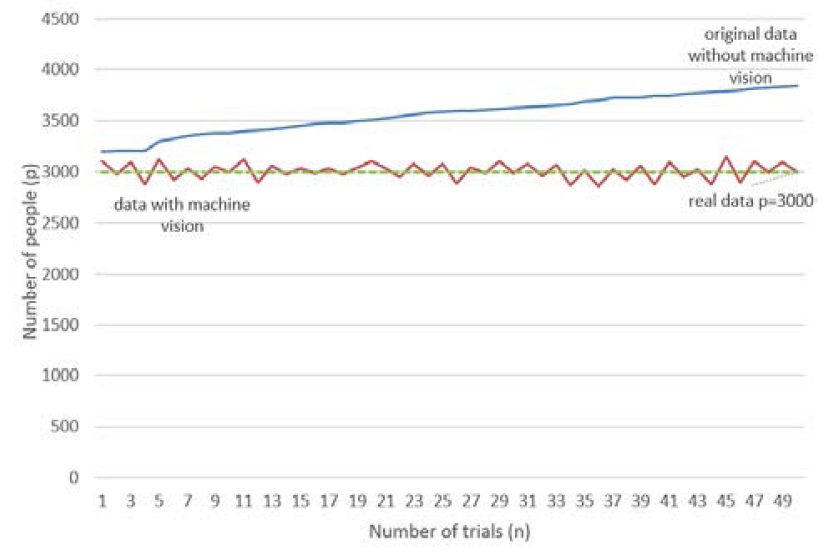

Fig. 4. Line chart of test results of two methods.

Calculated from experimental data, the MAE calculated by the closed-loop verification method proposed in this study is 66.62 , and the MAE calculated by the numerical analysis method of system stability based on Lyapunov theory is 563.48. Therefore, it can be explained that the closed-loop verification method of this study has a high accuracy rate for crowd counting, and has reached the goal of improving the accuracy of crowd counting. 


\section{Summary}

This study adopts the complementary model of theoretical numerical analysis and machine vision to propose a method for stability analysis and closed-loop verification of the crowd merging system. The numerical analysis method of system stability based on Lyapunov theory can realize rapid prediction of crowd stability. However, this method is difficult to avoid random errors in the initialization of density and speed parameters, and cumulative errors due to time iteration, which affect its application. To this end, this study draws on feedback control strategies such as using the convolutional neural network model in machine vision to identify pedestrian positions and estimate crowd density from video data with high accuracy and high precision to verify the current stability numerical analysis conclusions. The actual measurement data is used to update the initial parameters of the stability analysis model at the next moment, which eliminates the stage error of the Lyapunov analysis method. As a result, a complementary crowd merging system stability analysis and closed-loop verification method are obtained.

This research is financially supported by the National Natural Science Foundation of China (No.72074170).

\section{References}

1. Zhao Rongyong,Liu Qiong,Li Cuiling,Dong Daheng,Hu Qianshan,Ma Yunlong. Assessment model for the Stability Situation of Crowd Evacuation based on Thermodynamic Map[J]. Journal of Physics: Conference Series,2021,1732(1).

2. Helbing, Dirk, and Pratik Mukerji. "Crowd Disasters as Systemic Failures: Analysis of the Love Parade Disaster.” EPJ Data Science, vol. 1, no. 1, 2012, doi:10.1140/epjds7.

3. Dong Wang. Research and Application of Macroscopic Model for Evacuation of Crowds[D]. Shanghai:Tongji University. 2018.

4. Qianshan Hu. Stability Analysis of Panic Crowd Evacuation at T-shaped Street Junction[D]. Shanghai:Tongji University. 2019.

5. Wang Fuhua, Li Jun,Ding Libo,Wang Yetao,Song Zhanping. Construction stability analysis of large section highway tunnel portal section based on numerical analysis[J]. IOP Conference Series: Earth and Environmental Science,2021,643(1).

6. Wei Zhang,Yongjie Wang,Yanyan Liu,Jianghua Zhu. Deep convolution network for dense crowd counting[J]. IET Image Processing,2020,14(4).

7. Zhikang Zou,Yu Cheng,Xiaoye Qu,Shouling Ji,Xiaoxiao Guo,Pan Zhou. Attend to count: Crowd counting with adaptive capacity multi-scale $\mathrm{CNNs}[\mathrm{J}]$. Neurocomputing,2019,367.

8. Nguyen Phuong T.,Di Ruscio Davide,Pierantonio Alfonso,Di Rocco Juri,Iovino Ludovico. Convolutional neural networks for enhanced classification mechanisms of metamodels[J]. Journal of Systems and Software,2021,172.

9. RAHMALAN H, NIXON M, CARTER J. On Crowd Density Estimation for Surveillance[C]. The Institution of Engineering and Technology Conference on Crime and Security. London, England: IEEE Press, 2006: 540-545. 